\title{
Extrinsic and intrinsic factors influencing the emergence and return of Asian particolored bats Vespertilio sinensis
}

\author{
Lei Feng ${ }^{1}$, Hexuan Qin ${ }^{1}$, Jingjing $\mathrm{Li}^{2}$, Xin $\mathrm{Li}^{2}$, Jiang Feng ${ }^{1}$, and Tinglei Jiang ${ }^{1}$ \\ ${ }^{1}$ Northeast Normal University \\ ${ }^{2}$ Jilin Agricultural University
}

October 28, 2021

\begin{abstract}
The circadian rhythm is an adaptive biological process, allows organisms to anticipate daily environmental changes and implement appropriate strategies. Circadian rhythms play a crucial role in the health and survival of organisms. However, little is known concerning how intrinsic and extrinsic factors affect animal daily rhythms in the field, especially in nocturnal animals. Here, we investigated the emergence and return times of Vesperilio sinensis, and also integrated environmental conditions (temperature, humidity and light intensity) and biotic factors (reproductive status and predation risk) to determine causes of variation in the activity rhythms of the bats. We found that variation in the first emergence time, the mid-emergence time, and the final return time were distinct. The results demonstrated that the emergence and return times of bats were affected by light intensity, reproductive status, and predation risk in a relatively complex pattern. Light intensity had the greatest contribution to activity rhythms. Moreover, we first investigated the effects of actual predators on the activity rhythms of bats; the results showed that the mid-emergence time of bats was earlier as predators were hunting, but the final return time was later when predators were present. This challenges the traditional view that high predation risk leads to later emergence and earlier return. Finally, our results also highlighted the importance of higher energy demands during the lactation period in bats to variation in activity rhythms. These results improve our understanding of the patterns and causes of variation in activity rhythms in bats and other nocturnal animals.
\end{abstract}

Extrinsic and intrinsic factors influencing the emergence and return of Asian particolored bats Vespertilio sinensis

Lei Fenga,b $\mid$ Hexuan Qina,b $\mid$ Jingjing $\operatorname{Li}^{\mathrm{c}}\left|\mathrm{Xin} \mathrm{Li}^{\mathrm{c}}\right|$ Jiang Fenga, ${ }^{\mathrm{a}}{ }^{*} \mid$ Tinglei Jiang, ${ }^{\mathrm{a}, \mathrm{b}^{*}}$

${ }^{a}$ Jilin Provincial Key Laboratory of Animal Resource Conservation and Utilization, Northeast Normal University, Changchun, China;

${ }^{\mathrm{b}}$ Key Laboratory of Vegetation Ecology of Education Ministry, Institute of Grassland Science, Northeast Normal University, 2555 Jingyue Street, Changchun, 130117, China;

${ }^{\mathrm{c}}$ College of Animal Science and Technology, Jilin Agricultural University, Changchun, China

${ }^{*}$ Corresponding author: Jiang Feng and Tinglei Jiang

E-mail: fengj@nenu.edu.cn; jiangt1730@nenu.edu.cn

Jiang Feng: Telephone: 86-0431-85098097; Fax number: 86-0431-85098097

Tinglei Jiang: Telephone: 86-0431-85098097; Fax number: 86-0431-85098097

\section{ABSTRACT}


The circadian rhythm is an adaptive biological process, allows organisms to anticipate daily environmental changes and implement appropriate strategies. Circadian rhythms play a crucial role in the health and survival of organisms. However, little is known concerning how intrinsic and extrinsic factors affect animal daily rhythms in the field, especially in nocturnal animals. Here, we investigated the emergence and return times of Vesperilio sinensis, and also integrated environmental conditions (temperature, humidity and light intensity) and biotic factors (reproductive status and predation risk) to determine causes of variation in the activity rhythms of the bats. We found that variation in the first emergence time, the mid-emergence time, and the final return time were distinct. The results demonstrated that the emergence and return times of bats were affected by light intensity, reproductive status, and predation risk in a relatively complex pattern. Light intensity had the greatest contribution to activity rhythms. Moreover, we first investigated the effects of actual predators on the activity rhythms of bats; the results showed that the mid-emergence time of bats was earlier as predators were hunting, but the final return time was later when predators were present. This challenges the traditional view that high predation risk leads to later emergence and earlier return. Finally, our results also highlighted the importance of higher energy demands during the lactation period in bats to variation in activity rhythms. These results improve our understanding of the patterns and causes of variation in activity rhythms in bats and other nocturnal animals.

Keywords: circadian rhythm, abiotic factors, biotic factors, reproduction status, predation, bats

\section{INTRODUCTION}

Circadian rhythms are endogenous patterns in the activity of organisms over periods of about $24 \mathrm{~h}$, and they are universal, from prokaryotes to eukaryotes (Bell-Pedersen et al., 2005; McClung, 2000). It is commonly considered that circadian rhythms are adaptive biological processes that are crucial to the health and survival of organisms, as they allow organisms to anticipate the daily environmental changes and implement appropriate strategies (Caravaggi et al., 2018; Yerushalmi \& Green, 2009). It is well known that global climate change has disrupted the activity rhythms of many animals (Levy, Dayan, Porter, \& Kronfeld-Schor, 2018). Environmental conditions and biotic factors have been considered drivers of variation in the circadian activity rhythms of many vertebrates (Pita, Mira, \& Beja, 2011). However, little is known concerning how intrinsic and extrinsic factors influence animal daily rhythms in the field (Quaglietta, Mira, \& Boitani, 2018).

Activity rhythms are influenced by many abiotic and biotic factors, including light, moon phase, temperature, humidity, reproductive status, and predation risk (Tester \& Figala, 1990). Light is an important modulator of organisms' circadian rhythms (LeGates, Fernandez, \& Hattar, 2014). Bright light conditions may present a dangerous situation for nocturnal animals, and the opposite would hold for day-active animals. For example, some nocturnal animals may decrease their activity during a full moon; this is known as "lunar phobia" (Mougeot \& Bretagnolle, 2000; R. A. Saldaña-Vázquez \& Munguía-Rosas, 2013). Extreme weather conditions could affect animals' activity patterns to avoid overheating or hyperthermia, causing animals to reduce their activity during high- or low-temperature conditions (Foà \& Bertolucci, 2001; Frick et al., 2012; Speakman, Hays, \& Webb, 1994). Additionally, drought could affect animal and plant populations and constrain the activity of prey, thereby increasing the competition for food (Frick et al., 2012).

In addition to abiotic factors, biotic factors such as predation risk and reproductive status also may influence animals' activity rhythms (Svensson et al, 2018). Predation risk is a strong selection pressure affecting activity rhythms, and predator avoidance is a major topic in animal evolution (John, 2013; Lima \& O'Keefe, 2013). Animals face a tradeoff between predation risk and energy requirements when they decide to initiate activity during the period of day-to-night transition, especially for female animals during lactation, an energetically demanding period (Arndt., O'Keefe., Mitchell., Holmes., \& Lima., 2018; Shiel \& Fairley, 1999; Shuert, Halsey, Pomeroy, \& Twiss, 2020). Females must balance increases in foraging time while maximizing food availability and minimizing the possibility of being captured by predators (Caro, 2005). In general, an earlier start to foraging activity may be beneficial (Pavey, Burwell, Grunwald, Marshall, \& Neuweiler, 2001), but may also expose animals to a higher predation risk (Fenton et al., 1994; Jones \& Rydell, 1994). Thus, a successful strategy requires the optimal adjustment between predation pressure and foraging activity in order to maximize energy intake (Caro, 2005). However, it remains unclear how animals adjust activity rhythms 
based on predation risk and energy requirement, especially during the lactation period.

Bats are an excellent system for investigating questions concerning the effects of abiotic and biotic factors on activity rhythms for the following reasons. First, as one of the most abundant mammal groups in terms of species and ecological diversity, bats are active almost exclusively during the night (Rydell \& Speakman, 1995; Speakman, 1991). In this case, bats are sensitive to environmental changes, especially to light (Voigt \& Kingston, 2016). Second, bats normally start nighttime activity for foraging after sunset, but not in total darkness (Lee \& McCracken, 2001; Pavey et al., 2001). Like other animals, bats would benefit from an earlier emergence that would allow the bats to follow the activity peaks of some insects (i.e., Nematocera and other small flies) during dusk (Rydell, Entwistle, \& Racey, 1996). Moreover, although earlier onset of activity would increase the amount of foraging time at dusk and dawn (Pavey et al., 2001), it would increase the chance of bats encountering diurnal predators that remain active around sunset, or even during dusk (Fenton et al., 1994; Lima \& O'Keefe, 2013). However, this view was proposed based on an estimate of predation risk without actual predators. Thus, it is necessary to clarify the effects of natural predation and energy requirements for reproduction on the activity rhythms of bats. Finally, previous studies have focused on the effects of environmental factors such as temperature (Arndt. et al., 2018; Irwin \& Speakman, 2003), cloud cover (Arndt. et al., 2018; Lee \& McCracken, 2001; McAney \& Fairley, 1988), heavy rain (McAney \& Fairley, 1988) and light (Russo, LucaCistrone, \& GarethJones, 2007) on the emergence of bats, but little is known concerning the impact factors affecting the return times of bats, or how interactions of abiotic and biotic factors influence the activity rhythms of bats.

In this study we monitored the emergence and return times of Asian particolored bats (Vesperilio sinensis ), and integrated environmental conditions (temperature, humidity and light intensity) and biotic factors (reproductive status and predation risk) to determine the sources of variation in activity rhythms of bats. We tested three hypotheses. First, we hypothesized that light intensity may play a pivotal role in influencing the emergence and return times of $V$. sinensis. Second, since bats during the lactation period have greater energy requirements, we hypothesized that $V$. sinensis would depart from the roost earlier during the lactation period than during the post- lactation period. Third, since bats should avoid predators, we hypothesized that $V$. sinensis would depart from the roost at dusk later, but return to the roost earlier at dawn in the presence of diurnal predators (e.g., falcons).

\section{MATERIALS AND METHODS}

\section{Study site and species}

The study was conducted at a highway bridge (12657'26" E, 45deg32'52" N) in Acheng district, Heilongjiang Province, northeast China. A large number of $V$. sinensis roost under the bridge. In summer, female $V$. sinensis roost and breed offspring here, forming a nursery (Wang., BY Shi, X Zhao, Feng., \& Jiang., 2020). The population of bats arrives in the summer roost in the middle of June and leaves the summer roost by the end of September. We found thatFalco amurensis started to prey on $V$. sinensis during mid-August in 2018; this provided an opportunity to determine the effects of predation on activity rhythms of $V$. sinensis .

\section{Monitoring of activity rhythms}

Roost emergence time and final return time were recorded from the middle of July to early September, from 2019 to 2021. The monitoring was stopped when it was raining heavily. In this study, we arrived at the roost of bats one hour before sunset. The first emergence time was defined as the first bat to emerge and leave the roost. We also arrived at the roost of the bats an hour and a half before sunrise. The final return time was observed and recorded when the last bat returned to the day roost. Additionally, previous studies showed that median emergence time was a better parameter to describe the emergence time of the whole roost than first emergence (Bullock., Combes., Eales., \& Pritchard., 1987). Therefore, we also included the median emergence time as a descriptive parameter for emergence time of $V$. sinensis. We defined the median of the first emergence time and the end time of emergence as the mid-emergence time. In order to calculate the median time between the starting and ending of the emergence events, we recorded the data until the end of emergence. When there were no bats flying out for five minutes, we considered the emergence activities 
of the bats to have finished.

\section{Collection ofenvironmental data}

We recorded the sunrise and sunset times through the network https://richurimo.51240.com/ based on the longitude and latitude of the roost of $V$. sinensis . Here, the time was accurate to the nearest second. One researcher recorded the first emergence time and the final return time. Another researcher simultaneously measured the light intensity, air temperature, and humidity using a Five in One Multifunctional Environment Meter (HT-8500, Guangzhou, China). The environmental data were measured at the same location on the highway bridge without objects blocking natural light or external artificial light sources. In this study, the final return times were recorded in 2019 and 2021. Additionally, we recorded the light intensity of natural light every 30 seconds in 2019 and 2021. Then, we determined the light intensity of the median of emergence events by selecting the point in time closest to the median of emergence.

\section{The division of reproductive status}

A previous study showed that the lactation period of $V$. sinensisoccurs in July at this roost (Yin, 2020). In this study, in order to investigate the effects of reproductive state on activity rhythms of $V$. sinensis we divided the monitoring period into two periods: a lactation period (during July) and a post-lactation period (after July) based on our previous observations.

\section{Predation risk assessment}

In order to explore the effects of diurnal predators on the activity rhythms of $V$. sinensis, we defined predation risk as presence or absence of predators. If the predator $F$. amurensis appeared near the roost and hunted bats, this was considered as the presence of predators (high predation risk) when we were monitoring the first emergence and the final return. If there were no $F$. amurensis, or $F$. amurensis only flew across the roost without predation attempts, this was considered as absence of predators (low predation risk). Since $F$. amurensisarrived at the roost to hunt bats every day in summer, $F$. amurensis was present during mid-emergence. In this case, we considered predation risk as "predators were hunting" or "predators were not hunting" when determining mid-emergence time. During the monitoring period, we did not observe any other potential diurnal avian predator near the roost. Therefore, we believed that only F. amurensisexerted diurnal predation pressure on $V$. sinensis as they were flying out and returning.

\section{Data analysis}

In this study, we used differences between sunset time and first emergence time of bats, and between sunset time and mid-emergence time, as two variables to quantify the time of emergence of bats. Moreover, we also used differences between sunrise and final return time as a variable to quantify the return time of bats. The three variables were tested for normality by Shapiro-Wilk tests. We found that only differences between sunrise and final return time did not follow a normal distribution $(P<0.001)$. In this case, the logarithmic transformation was used to make the data meet a normal distribution $(P=0.407)$.

Here, in order to state and use statistics more easily, we use the following abbreviations for independent variables measured at dusk, which were used to construct models to test the effects of these variables on emergence times of bats: HFE, Humidity at first emergence of bats; HME, Humidity at median emergence of bats; TFE, Temperature at first emergence of bats; TME, Temperature at median emergence of bats; SST, Sunset time; LISS, Light intensity of sunset; LIFE, light intensity of first emergence; LS, Lactation stages (lactation or post-lactation); PAPDD, Presence or absence of predators during dusk; LME, light intensity of mid-emergence; PHNHM, Predators were hunting or not hunting in mid-emergence. Additionally, variables measured at dawn were considered independent variables for the analysis of the effects of factors on final return events: HFR, Humidity at final return of bats; TFR, Temperature at final return of bats; SRT, Sunrise time; LISR, Light intensity at sunrise; LS, Lactation stages (lactation or post-lactation); PAPDA, Presence or absence of predators during dawn; LIFR, Light intensity at final return.

To test the effects of environmental factors, reproduction, and predation on the emergence time of $V$. 
sinensis, we selected optimized linear models using the dredge function in the package "MuMin" in R v. 3 . 5.1 (R Core Development Team, 2018). In first model, we used the difference between sunset time and first emergence time of bats as a dependent variable; HFE, TFE, SST, LISS, LS, PAPDD and LIFE as independent variables; and year as a random variable. In the second model, we used the difference between sunset time and mid-emergence time as a dependent variable; HME, TME, ST, LISS, LIFE, LIME, LS and PHNHM as independent variables; and year as a random variable. Additionally, to test the effects of environmental factors, reproduction, and predation on the final return time of $V$. sinensis, we also selected optimized linear models using the dredge function in the package "MuMin" in $\mathrm{R} \mathrm{v}$. 3.5.1. In the third model, we used the difference between sunrise time and final return time as a dependent variable; HFR, TFR, SRT, LISR, LS, PAPDA and LIFR as independent variables; and year as a random variable. To test the multicollinearity of variables, the Variance Inflation Factor (VIF) of each predicted factor was calculated to determine which predictors could not be used for subsequent analysis. If $\mathrm{VIF}$ was $<5$, the corresponding predictor variables were included in the models (Lin. \& Feng, 2008). We compared the models using the Akaike information criterion corrected for small sample size (AICc). The model with the lowest AICc indicates the best-fitting model. We caculated $\triangle \mathrm{AICc}$ as the difference of AICc of each model between the AICc of the best-fitting model. A difference of AICc $>2(\Delta$ AICc $>2)$ indicated that the model with the lower AIC value had better explanatory power (Anderson \& Burnham, 2002). If the $\Delta \mathrm{AICc}$ was [?] 2, multimodel inference was performed using the function model.avg in the package 'MuMIn' (Bartoń., 2014) in R v. 3.5.1. We also calculated Akaike weights $(W i)$ to assess the relative likehood of a model compared with other models. Additionally, we conducted a hierarchical partitioning analysis in the 'hier.part package' (Walsh. \& Nally., 2020) in R v. 3.5.1 to estimate the independent effect of each predictor variable (Chevan. \& Sutherland., 1991).

Multiple linear regression analyses were used to assess the relationships between sunset time and first emergence time and LIFE, between sunset time and first emergence time and LISS, between between sunset time and mid-emergence time and LIFE, between between sunset time and mid-emergence time and LISS, between between sunrise and final return time and LIFR, and between sunrise and final return time and LISR. During the analysis, if the relationships between dependent variables and independent variables were better simulated by a curve, a curvilinear regression analysis was performed. In this study, we used curvilinear regression analyses to assess the relationships between sunset time and first emergence time and the variables LIFE, between sunset time and mid-emergence time and LIFE, and between sunrise and final return time and LIFR. All statistical analyses were conducted in R v. 3.5.1.

\section{RESULTS}

\section{The impact factors of first emergence time of $V$. sinensis}

A total of 140 first emergence events of $V$. sinensis were recorded. There were no significant differences in first emergence time among the three consecutive years (Kruskal-Wallis test: $H=2.804, P=0.246$ ). In 49 records, $V$. sinensis started to emerge before sunset at dusk. Differences between sunset and first emergence ranged from -36 to $33 \mathrm{~min}(4.49 \pm 13.23)$. Here, the negative values meant that first emergence events started before sunset.

Our analyses indicated that the best model describing the variation in first emergence time of $V$. sinensis used LIFE, LISS, and LS as the predictor variables (Table 1). Further, model averaging revealed that the three predictive variables were also significantly associated with the first emergence time of $V$. sinensis (Table 2 ). Differences between first emergence time and sunset time were significantly and negatively associated with light intensity of first emergence (linear regression: $t=-11.447, P<0.001$, Fig. 1b; curvilinear regression: $t$ $=13.728, P<0.001$, Fig. 5a). In other words, the higher the light intensity at first emergence, the earlier the first emergence of $V$. sinensis. Moreover, differences between first emergence time and sunset time were significantly and positively associated with light intensity at sunset $(t=7.801, P<0.001$; Fig. 1a). Finally, significant differences in the first emergence time were detected between lactation and post-lactation $(t=$ $-4.779, P<0.001 ;$ Fig. 1c). During the lactation period, $V$. sinensis departed earlier from the roost than during the post-lactation period (Fig. 1c). Specifically, $65.67 \%$ of first emergence events $(44 / 67)$ started 
before sunset during the lactation period. In contrast, only $6.85 \%$ of first emergence events (5/73) were observed before sunset during the post-lactation period. A Chi-square test of independence showed that the first emergence events had a higher probability of occuring before sunset during the lactation period $\left(\chi^{2}=\right.$ $\left.26.34, P=2.86 \times 10^{-7}\right)$. Consistent with the results of the hierarchical partitioning analysis, the independent contributions of LIFE, LISS, and LS were $53.86 \%, 25.54 \%$, and $14.17 \%$, respectively (Fig. 4a).

\section{The impact factors of the mid-emergence time of $V$. sinensis}

We recorded 91 mid-emergence events of $V$. sinensis, only two events occurred before sunset. The difference between mid-emergence time and sunset ranged from -4 to $62 \mathrm{~min}$. Here, the positive values meant midemergence events happened after sunset. The light intensity of mid-emergence events was approximately 0.6-400 lux (40.46+-68.72). In addition, 90.11\% (82/91) mid-emergence events occurred when the light intensity was below 100 lux, and $45.05 \%$ (41/91) of mid-emergence events occurred when the light intensity was below 10 lux.

Our analyses indicated that the best model of variation in mid-emergence time of $V$. sinensis used LIFE, LISS, LS, and PHNHM as the predictor variables (Table 3). Further, model averaging revealed that the four predictor variables were also significantly associated with the mid-emergence time of $V$. sinensis (Table 4). First, differences between the mid-emergence time and sunset time were significantly and negatively associated with light intensity of first emergence (linear regression: $t=-6.73, P<0.001$, Fig. 2b; curvilinear regression: $t=-9.974, P<0.001$, Fig. $5 \mathrm{~b}$ ). That is, the higher the light intensity of first emergence, the earlier the mid-emergence time of $V$. sinensis. Second, a significant and positive correlation between the mid-emergence time and light intensity at sunset was observed $(t=5.882, P<0.001$; Fig. 2a). Third, a significant difference in the mid-emergence time was detected between lactation and post-lactation $(t=$ -2.903, $P=0.004$; Fig. 2d). During the lactation period, $V$. sinensis departed earlier from the roost than during the post-lactation period (Fig. 2d). Specifically, the mid-emergence events started approximately -4-40 (15.435+-10.051) min after sunset during the lactation period. In contrast, the mid-emergence events started about 12-62 (29.511+-9.201) minutes after sunset during the post-lactation period. Finally, bats also departed from the roost earlier when predators were hunting $(t=-3.713, P<0.001$; Fig. $2 \mathrm{c})$. We found that predators were hunting bats during 36 mid-emergence events. Consistent with the results of the hierarchical partitioning analysis, the independent contributions of LIFE, LISS, LS, and PHNHM were 46.41\%, 13.60\%, $22.74 \%$ and $17.25 \%$, respectively (Fig. $4 \mathrm{~b}$ ).

\section{The impact factors for the final return time of $V$. sinensis}

We recorded 78 final return events of $V$. sinensis, and all final return events occurred and ended approximately 7-79 minutes before sunrise. There were no significant differences in the final return time between 2019 and 2020 (Paired sample t test, $t=1.730, P=0.093$ ).

Our analyses indicated that the best model of variation in the final return time of $V$. sinensis used LIFR and PAPDA as the predictor variables (Table 5). However, model averaging revealed that three predictive variables (LIFR, LISR, and PAPDA) were also significantly associated with the final return time of $V$. sinensis (Table 6). First, differences between the final return time and sunrise time were significantly and positively associated with light intensity at sunrise $(t=4.054, P<0.001$; Fig. 3a). That is, the higher the light intensity at sunrise, the earlier the final return time of $V$. sinensis. Second, differences between sunrise and final return time were significantly and negatively associated with light intensity at final return (linear regression: $t=-8.194, P<0.001$, Fig. 3b; curvilinear regression: $t=-10.226, P<0.001$, Fig. 5c). Finally, bats also returned to the roost later when predators were present $(t=-5.746, P<0.001$; Fig. $3 \mathrm{c})$. We found that predators were often (60\% records at dawn) present before the ending of the final return events at dawn. However, the number of successful predation events occurring at dawn was significantly less than at dusk (Mann-Whitney $U$ test, $\mathrm{Z}=-1.993, P=0.046$ ). Consistent with the results of the hierarchical partitioning analysis, the independent contributions of LIFR, LISR, and PAPDA were $56.34 \%, 6.18 \%$, and $37.49 \%$, respectively (Fig. 4c).

\section{DISCUSSION}


In our study, we found that light intensity at emergence, return, sunset, andd sunrise influenced the emergence and return times of $V$. sinensis in different ways, supporting our first hypothesis. Moreover, $V$. sinensis departed from the roost earlier regardless of the first emergence and mid-emergence times during the lactation period, suggesting support for our second hypothesis. Finally, the mid-emergence time of $V$. sinensis was earlier when predators were hunting, but the final return time was later when predators were present. This result was inconsistent with our third hypothesis.

Factors affecting an animal's energy status and the value of additional energy intake are major determinants of risk-taking behaviour (Brown, 1988; Caro, 2005; Lima, 1998). In general, the first emergence of bats occurs after sunset (Acharya, Racey, McNeil, Sotthibandhu, \& Bumrungsri, 2015; Arndt. et al., 2018; Lee \& McCracken, 2001; Welbergen, 2006) and return events occur before sunrise (Acharya et al., 2015; Lee \& McCracken, 2001). During lactation, reproductive female bats would emerge before sunset because of the higher energy demand (Arndt. et al., 2018; Lee \& McCracken, 2001). This behaviour has been noted in hoary bats, Lasiurus cinereus(Barclay, 1989), Mexican free-tailed bats, Tadarida brasiliensis(Lee \& McCracken, 2001), the grey-headed flying fox, Pteropus poliocephalus(Welbergen, 2006), Daubenton's bats,Myotis daubentonii (Lučan, 2009), dawn nectar bats, Eonycteris spelaea(Acharya et al., 2015), and Indiana bats, Myotis sodalist(Arndt. et al., 2018). Additionally, during lactation, reproductive female bats also return later to the day roost than during post-lactation, such as in T. brasiliensis (Lee \& McCracken, 2001) and Dawn nectar bats, E . spelaea (Acharya et al., 2015). Consistent with these previous studies, our results showed that the lactation period significantly influenced the first emergence and the mid-emergence of $V$. sinensis during the lactation period. Specifically, $64.29 \%$ of emergence events started before sunset during the lactation period, while only $5.88 \%$ of emergence events started before sunset during the postlactation period. The earlier onset of activity and the delay of ending activity increases the foraging time at dusk and dawn (Rydell et al., 1996). To summarize, the higher energy demands may prompt bats to depart from the roost earlier and return to the roost later during the lactation period.

There are many benefits to an early start to night activity, but for bats, doing so in bright light may be a risky option. Large flocks of bats emerging from the day roost and returning to the day roost would attract the attention of diurnal avian predators (Fenton et al., 1994). Predation on bats by birds is seemingly rare and opportunistic at dusk and dawn, especially in temperate zones, but predation would affect many aspects of bats' behaviour, such as roost selection and foraging activity (Fenton et al., 1994; Lima \& O'Keefe, 2013). Bats emerging around dusk before sunset under high light intensity would be exposed to higher predation risk than the bats emerging in true night under low light intensity. As for earlier emerging bats, later returning bats also would be exposed to high predation risk from diurnal predators (Fenton et al., 1994). However, previous studies have mainly assessed predation risk without observing real predators. In this case, the effects of predation on activity rhythms of bats may not be universal. In this study, we found that the mid-emergence time of $V$. sinensis was earlier when predators were hunting, but the final return time was later when predators were present. The results may challenge the previous view that high predation risk leads to later emergence and the earlier return. This can be explained by the following reasons: First, the previous studies investigated the effects of predation risk on behaviour of bats based on empirical estimation or predator models, but here $F$. amurensis hunt $V$. sinensis every day at this roost. Thus, the different results may not be inconsistent. Second, $V$. sinensis usually gives birth to twins in each litter (Jin. et al., 2012), and the energy demands are greater during the lactation period than in other periods or in bat species with one offspring per litter. Thus, even though $V$. sinensis may suffer higher risk of predation, they may need to depart from the roost earlier and return to the roost later during the lactation period due to the higher energy demands. The hierarchical partitioning analysis also confirmed that lactation periods had a greater contribution to the mid-emergence time and the final return time in $V$. sinensis than predation risk. Interestingly, predation risk only influenced the mid-emergence time rather than the first emergence time in $V$. sinensis. This may be because $V$. sinensis had to depart in quantity around the mid-emergence time due to $F$. amurensis successfully preying on bats in dim light (light intensity below 10 lux); this also may reduce the risk of predation on individuals via the dilution effect of a large flock of bats.

Bright light conditions may present a risky option for nocturnal animals, especially for bats. In this study, 
light intensity was the most important factor affecting the activity rhythm of $V$. sinensis $\mathrm{t}$ (Fig. 4). Specifically, differences between first emergence time and sunset time, and between mid-emergence time and sunset time, were significantly and positively associated with light intensity at sunset (Fig. 1a; Fig. 2a). A similar trend was detected in the relationship between final return time and sunrise (Fig. 3a). These results showed that $V$. sinensis may adjust the emergence and return times based on sunset and sunrise times, respectively. This may be helpful for avoiding $F$. amurensis for the following reasons. With the decrease of light intensity, the visual sensitivity of falcons decreases significantly (Fox., Lehmkuhle., \& Westendorf., 1976). Therefore, it was clearly a safer behavioural strategy for bats to emerge from the roost under dim light conditions. Additionally, bright light exerts inhibitory effects on the activity of bats (A.Gutierrez, F.Pessoa, Aguiar, \& M.A.Pesso, 2014). However, here the opposite trends of the effect of light intensity of first emergence on the emergence time (Fig. 1b; Fig. 2b) and of light intensity at final return on the final return time (Fig. 3b) were observed. With increases in light intensity of first emergence, both the first emergence and midemergence events became increasingly earlier relative to sunset, then changed slowly (Fig. 5a, b). Moreover, with increases in light intensity at final return, the final return events became increasingly later relative to sunrise, then changed slowly (Fig. 5c). These findings may be due to the high energy demands in V. sinensis during the lactation period prompting the bats to depart from the roost earlier and return to the roost later despite the high light intensity. We observed that $65.67 \%$ of first emergence events (44/67) started before sunset during the lactation period, supporting this view. Additionally, there is a threshold of light intensity in bat activity rhythms (Erkert, 2004), which may reflect the fact that leaving the day roost early at dusk or returning to the roost late at dawn was not the best option for the bats.

Weather conditions may also affect the emergence behaviour of bats, but the effects in the previous studies were not severe (Erickson \& West, 2002; Frick et al., 2012; Welbergen, 2008). For example, the relationship between temperature and the start of emergence of bats depended on summer climatic conditions; therefore, the influence of daily temperature in drought conditions may be different than in normal or unusually moist years (Frick et al., 2012). Below a critical minimum temperature $\left(8-10^{\circ} \mathrm{C}\right)$, the foraging behaviour of bats would be less beneficial than remaining torpid (Avery, 1985). Drought conditions were associated with low insect abundance (Hawkins et al, 1998), and thus were associated with earlier emergence and displaying risker behaviour (Frick et al., 2012). Consistent with these previous studies, our results indicated that temperature and humidity did not influence emergence or return times of $V$. sinensis. This may be because environmental temperatures at the roost of $V$. sinensis were higher than the critical minimum temperature during the monitoring period, and there was sufficient rainfall in summer. The relatively stable environment led to the relatively high insect abundance. In this case, the effects of temperature and humidity on activity rhythms of bats may be relatively weak.

\section{CONCLUSIONS}

In summary, our study demonstrated that the emergence and return times of $V$. sinensis were affected by light intensity, reproductive status, and predation risk in a relatively complex pattern, indicating that the decisions concerning emergence and return of $V$. sinensishad a high degree of plasticity. Therefore, our study investigated the effects of real predators on activity rhythms of bats, and the results challenged the traditional view that high predation risk leads to the later emergence and earlier return. Our results also highlighted that higher energy demands during lactation period in bats may be more important than predation risk in the variation in activity rhythms. A limitation of this study is that it is difficult to determine how $V$. sinensis make decisions of emergence and return based on the intrinsic and extrinsic factors according to our present data. Further studies will need to determine the causal links between activity rhythms of bats and their impact factors.

\section{ACKNOWLEDGEMENTS}

We are grateful to Liu Yingying, Zhao Xin and Wang Yuze for their help during field work. We thank LetPub (www.letpub.com) for its linguistic assistance during the preparation of this manuscript.

This research was supported by the National Natural Science Foundation of China (Grant Nos. 31922050, 
31872680, 32071492).

\section{CONFLICT OF INTEREST}

The authors declare that they have no conflict of interest.

\section{AUTHOR CONTRIBUTIONS}

Lei Feng: Conceptualization (equal); Data curation (equal); Formal analysis (lead); Investigation (equal); Methodology (lead); Visualization (lead); Writing-original draft (lead). Hexuan Qin: Investigation (equal), Jingjing Li: Investigation (equal), Xin Li: Investigation (equal), Jiang Feng:Funding acquisition (equal); Resources (lead). Tinglei Jiang:Conceptualization (equal); Data curation (equal); Funding acquisition (equal); Project administration (lead); Supervision (lead); Writing-review and editing (lead)..

\section{DATA AVAILABILITY STATEMENT}

If our paper is accepted for publication, data used in this study will be archived on the Dryad Digital Repository.

Table 1 Candidate linear mixed models explaining the variation in first emergence time of Vespertilio sinensis based on environmental parameters (temperature, relative humidity and light intensity) and biotic factors (reproductive stages and predation risk) at dusk.

\begin{tabular}{llccccc}
\hline Model & Predictive variables & $k$ & $d f$ & Loglik & ${ }^{A} I C c$ & $w i$ \\
\hline M1 & LIFE(-), PAPDD(+), LS(-), LISS(+) & 4 & 7 & -459.748 & 0.000 & 0.581 \\
M2 & LIFE(-), LS(-), LISS(+) & 3 & 6 & -462.302 & 2.890 & 0.137 \\
M3 & LIFE(-), TFE(+), PAPDD(+), LS(-), LISS(+) & 5 & 8 & -460.326 & 3.406 & 0.106 \\
M4 & HFE(+), LIFE(-), PAPDD(+), LS(-), LISS(+) & 5 & 8 & -460.347 & 3.448 & 0.104 \\
M5 & HFE(+), LIFE(-), LS(-), LISS(+) & 4 & 7 & -462.817 & 6.138 & 0.027 \\
M6 & LIFE(-), LS(-), TFE(+), LISS(+) & 4 & 7 & -462.962 & 6.427 & 0.023 \\
M7 & HFE(+), LIFE(-), TFE(+), PAPDD(+), LS(-),LISS(+) & 6 & 9 & -460.981 & 7.003 & 0.018 \\
M8 & HFE(+), LIFE(-), TFE(+), LS(-),LISS(+) & 5 & 8 & -463.413 & 9.580 & 0.005 \\
M9 & LIFE(-), PAPDD(+), LISS(+) & 3 & 6 & -471.697 & 21.681 & 0.000 \\
M10 & LIFE(-), TFE(+), PAPDD(+), LISS(+) & 4 & 7 & -471.309 & 23.122 & 0.000 \\
\hline
\end{tabular}

Models are ranked by Akaike' s Information Criterion corrected for small sample sizes (AICc) values, from the best to the worst model. LIFE: light intensity of first emergence of bats, LISS: light intensity of sunset, HFE: humidity as first emergence of bats, TFE: temperature as first emergence of bats, LS: lactation stages, PAPDD: presence or absence of predators during dusk.

Table 2 Model-averaged parameter estimates of the best-supported (before and including the null model) linear mixed models describing the variation in first emergence time of Vespertilio sinensis. The independent effects (IF) of factors on the first emergence time of Vespertilio sinensis using hierarchical partitioning analysis are displayed in the last column.

\begin{tabular}{llllllll}
\hline Variable & Estimate & $\mathrm{SE}$ & $\mathrm{Z}$ value & $\mathrm{t}$ value & $p$ & $95 \% \mathrm{CI}$ & $\mathrm{IF}$ \\
\hline (Intercept) & 5.436 & 1.943 & 2.775 & 3.060 & 0.005 & $1.597,9.274$ & $/$ \\
LIFE & -0.020 & 0.002 & 11.312 & -11.447 & $<0.001$ & $\mathbf{- 0 . 0 2 4}, \mathbf{- 0 . 0 1 7}$ & 53.860 \\
PAPDD & -2.267 & 1.408 & 1.596 & -1.602 & 0.110 & $-5.052,0.517$ & 3.436 \\
LS & -7.466 & 1.550 & 4.773 & -4.779 & $<0.001$ & $\mathbf{- 1 0 . 5 3 2 , - 4 . 4 0 1}$ & 14.168 \\
LISS & 0.030 & 0.004 & 7.741 & 7.801 & $<0.001$ & $\mathbf{0 . 0 2 2}, \mathbf{0 . 0 3 8}$ & 25.536 \\
\hline
\end{tabular}


Table 3 Candidate linear mixed models explaining the variation in mid-emergence time of Vespertilio sinensis based on environmental factors (temperature, relative humidity, and light intensity), and biotic factors (reproductive stages and predation risk) at dusk.

\begin{tabular}{lllllll}
\hline Model & Predictive variables & $k$ & $d f$ & Loglik & ${ }^{A} I C c$ & $w i$ \\
\hline M1 & LIFE(-), PHNHM (+), LS(-), LISS(+) & 4 & 7 & -300.237 & 0.000 & 0.674 \\
M2 & LIFE(-), PHNHM (+), TME(-), LS(-), LISS(+) & 5 & 8 & -300.818 & 3.569 & 0.113 \\
M3 & LIFE(-), PHNHM (+), LISS(+) & 5 & 6 & -304.161 & 5.499 & 0.043 \\
M4 & LIFE(-), PHNHM (+), LS(-), LISS(+), ST(-) & 4 & 8 & -301.814 & 5.561 & 0.042 \\
M5 & LIFE(-), LIME(-), PHNHM (+), LS(-), LISS(+) & 5 & 8 & -302.01 & 5.952 & 0.034 \\
M6 & LIFE(-), PHNHM (+), TME(-), LISS(+) & 6 & 7 & -303.849 & 7.225 & 0.018 \\
M7 & LIME(-), PHNHM (+), LS(-), LISS(+) & 3 & 7 & -303.862 & 7.251 & 0.018 \\
M8 & LIFE(-), PHNHM (+), LISS(+), ST(-) & 4 & 7 & -303.993 & 7.511 & 0.016 \\
M9 & LIFE(-), HME(+), PHNHM (+), LS(-), LISS(+) & 5 & 8 & -303.458 & 8.849 & 0.008 \\
M10 & LIFE(-), PHNHM (+), TME(-), LS(-), LISS(+), ST(-) & 5 & 9 & -302.461 & 9.320 & 0.006 \\
M11 & LIFE(-), LIME(-), PHNHM (+), TME(-), LS(-), LISS(+) & 4 & 9 & -302.616 & 9.631 & 0.005 \\
M12 & LIME(-), PHNHM (+), TME(-), LS(-), LISS(+) & 6 & 8 & -304.472 & 10.876 & 0.003 \\
M13 & LIFE(-), PHNHM (+), TME(-), LISS(+), ST(-) & 6 & 8 & -304.528 & 10.988 & 0.003 \\
M14 & LIFE(-), LIME(-), PHNHM (+), LS(-), LISS(+), ST(-) & 5 & 9 & -303.599 & 11.596 & 0.002 \\
M15 & LIME(-), PHNHM (+), LS(-), LISS(+), ST(-) & 5 & 8 & -305.05 & 12.033 & 0.002 \\
M16 & LIFE(-), LIME(-), PHNHM (+), LISS(+) & 4 & 7 & -306.356 & 12.239 & 0.001 \\
M17 & LIFE(-), HME(+), PHNHM (+), TME(-), LS(-), LISS(+) & 6 & 9 & -304.037 & 12.473 & 0.001 \\
M18 & LIFE(-), PHNHM (+), LS(-), ST(-) & 4 & 7 & -306.824 & 13.175 & 0.001 \\
M19 & LIFE(-), LS(-), LISS(+) & 3 & 6 & -308.228 & 13.633 & 0.001 \\
M20 & LIFE(-), LIME(-), PHNHM (+), LISS(+), ST(-) & 5 & 8 & -305.894 & 13.720 & 0.001 \\
M21 & LIFE(-), LIME(-), PHNHM (+), TME(-), LISS(+) & 5 & 8 & -305.97 & 13.873 & 0.001 \\
M22 & LIME(-), PHNHM (+), LS(-), ST(-) & 4 & 7 & -307.333 & 14.192 & 0.001 \\
M23 & LIFE(-), HME(+), PHNHM (+), LISS(+) & 4 & 7 & -307.359 & 14.244 & 0.001 \\
M24 & LIFE(-), HME(+), PHNHM (+), LS(-), LISS(+), ST(-) & 6 & 9 & -305.016 & 14.431 & 0.000 \\
\hline
\end{tabular}

Models are ranked by Akaike' s Information Criterion corrected for small sample sizes (AICc) values, from the best to the worst model. LIFE: light intensity of first emergence of bats, LISS: light intensity of sunset, LIME: light intensity of mid-emergence of bats, HME: humidity as mid-emergence of bats, TME: temperature at mid-emergence of bats, LS: lactation stages, PHNHM: predators were hunting or not hunting on bats at mid-emergence of bats, ST: sunset time.

Table 4 Model-averaged parameter estimates of the best-supported (before and including the null model) linear mixed models describing the variation in mid-emergence time of Vespertilio sinensis. The independent effects (IF) of factors on the mid-emergence time of Vespertilio sinensis using hierarchical partitioning analysis are displayed in the last column.

\begin{tabular}{llllllll}
\hline Variable & Estimate & $\mathrm{SE}$ & $\mathrm{Z}$ value & $\mathrm{t}$ value & $p$ & $95 \% \mathrm{CI}$ & $\mathrm{IF}$ \\
\hline (Intercept) & 20.365 & 2.750 & 7.299 & 7.440 & $<0.001$ & $14.896,25.833$ & $/$ \\
LIFE & -0.016 & 0.002 & 6.519 & -6.730 & $<0.001$ & $\mathbf{- 0 . 0 2 0 , - 0 . 0 1 1}$ & 46.410 \\
PHNHM & -5.758 & 1.555 & 3.650 & -3.713 & $<0.001$ & $\mathbf{- 8 . 8 4 9 , - 2 . 6 6 6}$ & 17.251 \\
LS & -4.828 & 1.665 & 2.859 & -2.903 & 0.004 & $\mathbf{- 8 . 1 3 8 , - 1 . 1 5 2}$ & 22.736 \\
LISS & 0.033 & 0.006 & 5.793 & 5.882 & $<0.001$ & $\mathbf{0 . 0 2 2 , \mathbf { 0 . 0 4 4 }}$ & 13.603 \\
\hline
\end{tabular}

Table 5 Candidate linear mixed models explaining the variation in final return time of Vespertilio sinen- 
sis based on environmental factors (temperature, relative humidity and light intensity) and biotic factors (reproductive stages and predation risk) at dawn.

\begin{tabular}{lllllll}
\hline Model & Predictive variables & $k$ & $d f$ & Loglik & ${ }^{A} I C c$ & $w i$ \\
\hline M1 & LIFR(+), PAPDA(-) & 2 & 5 & 45.617 & 0.000 & 0.552 \\
M2 & LIFR(+), PAPDA(-), LISR(+) & 3 & 6 & 46.432 & 0.720 & 0.385 \\
M3 & LIFR(+),TFR(-), PAPDA(-) & 3 & 6 & 43.726 & 6.132 & 0.026 \\
M4 & LIFR(+), LS(-), PAPDA(-) & 3 & 6 & 43.334 & 6.916 & 0.017 \\
M5 & LIFR(+), PAPDA(-), LS(-), LISR(+) & 4 & 7 & 44.294 & 7.412 & 0.014 \\
M6 & HFR(+), LIFR(+), PAPDA(-) & 3 & 6 & 41.256 & 11.072 & 0.002 \\
M7 & LIFR(+), TFR(-), PAPDA(-), LISR(+) & 4 & 7 & 42.361 & 11.278 & 0.002 \\
M8 & LIFR(+), TFR(-), PAPDA(-), LS(-) & 4 & 7 & 41.394 & 13.213 & 0.001 \\
M9 & LIFR(+), TFR(-), PAPDA(-), LS(-) & 4 & 7 & 41.134 & 13.732 & 0.001 \\
M10 & HFR(+), LIFR(+), PAPDA(-), LS(-) & 4 & 7 & 38.897 & 18.207 & 0.000 \\
\hline
\end{tabular}

Models are ranked by Akaike's Information Criterion corrected for small sample sizes (AICc) values, from the best to the worst model. LIFR: light intensity at final return of bats, LISR: light intensity of sunrise, HFR: humidity at final return of bats, TFR: temperature at final return of bats, LS: lactation stages, PAPDA: presence or absence of predators during dawn.

Table 6 Model-averaged parameter estimates of the best-supported (before and including the null model) linear mixed models describing the variation in final return time of Vespertilio sinensis. The independent effects (IF) of factors on the final return time of Vespertilio sinensis using hierarchical partitioning analysis are displayed in the last column.

\begin{tabular}{llllllll}
\hline Variable & Estimate & $\mathrm{SE}$ & $Z$ & $\mathrm{t}$ value & $p$ & $95 \% \mathrm{CI}$ & $\mathrm{IF}$ \\
\hline (Intercept) & $1.637 \mathrm{e}+0$ & $8.400 \mathrm{e}-2$ & 19.489 & 9.846 & $<0.001$ & $\mathbf{( 1 . 4 8 5 , \mathbf { 1 . 7 7 7 } )}$ & $/$ \\
LIFR & $-4.011 \mathrm{e}-3$ & $4.429 \mathrm{e}-4$ & 9.056 & -8.194 & $<0.001$ & $\mathbf{( - 0 . 0 0 5 , - 0 . 0 0 3 )}$ & 56.336 \\
PAPDA & $-2.062 \mathrm{e}-1$ & $3.439 \mathrm{e}-2$ & 5.996 & -5.746 & $<0.001$ & $\mathbf{( - 0 . 2 7 4 , - 0 . 1 4 1 )}$ & 37.488 \\
LISR & $3.604 \mathrm{e}-4$ & $7.627 \mathrm{e}-5$ & 4.726 & 4.054 & $<0.001$ & $\mathbf{( 0 . 0 0 0 , \mathbf { 0 . 0 0 1 ) }}$ & 6.177 \\
\hline
\end{tabular}
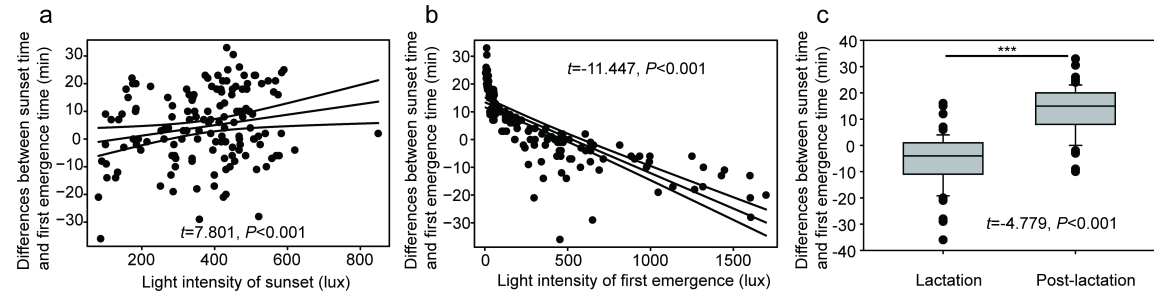

Figure 1 The relationships between differences (between sunset time and first emergence time) and light density of sunset (a), and light density at first emergence (b), and reproductive status (c), ${ }^{* * *}$ means $P$ $<0.001$. 

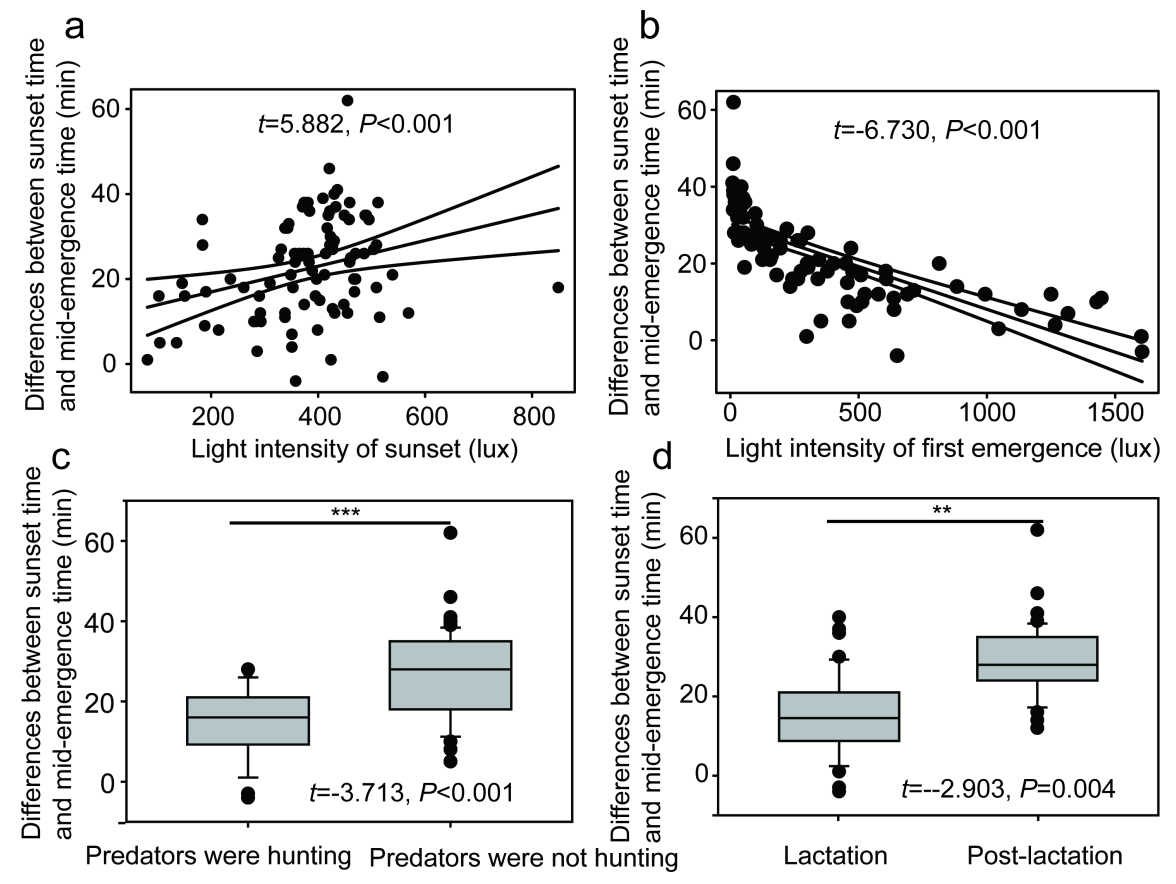

Figure 2 The relationships between differences (between sunset time and mid-emergence time) and light density of sunset (a), light density at first emergence (b), predation status (c) and reproductive status (d). ****means $P<0.001$.
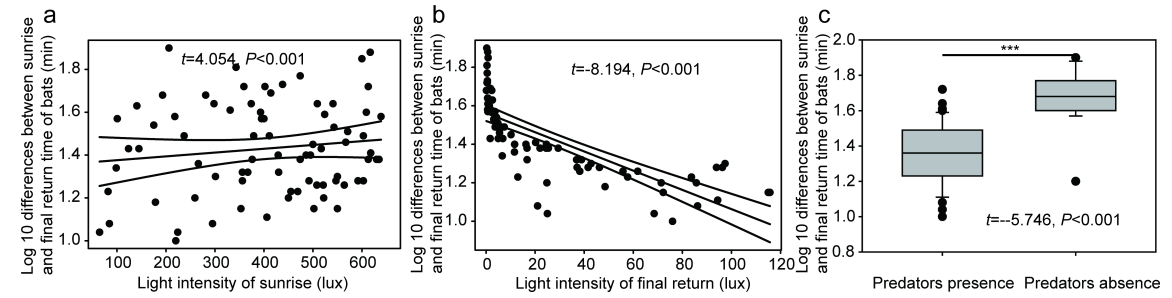

Figure 3 The relationships between differences (between final return time and sunrise time) and light density of sunrise (a), light density at final return (b), and predation status (c). $* * *$ means $P<0.001$.
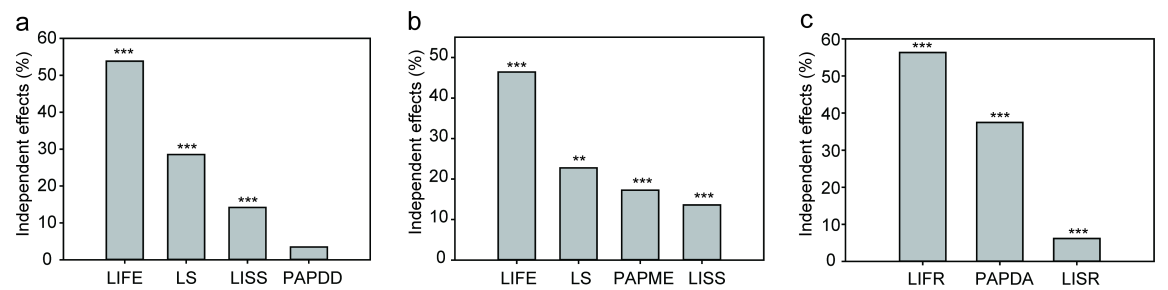

Figure 4 The independent contribution (\%) of each predictor calculated with hierarchical partitioning. ${ }^{* * *} P$ $<0.001$. (a): effects of predictor variables on the first emergence time, (b): effects of predictor variables on the mid-emergence time, (c): effects of predictor variables on the final return time. LIFE: light intensity of first emergence, LISS: light intensity of sunset, LS: lactation stage (lactation or post-lactation), PAPDD: presence or absence of predators during dusk, PHNHM: predation risk stages in mid-emergence, LIFR: light 
intensity at final return, PAPDA: presence or absence of predators during dawn, LISR: light intensity at sunrise.
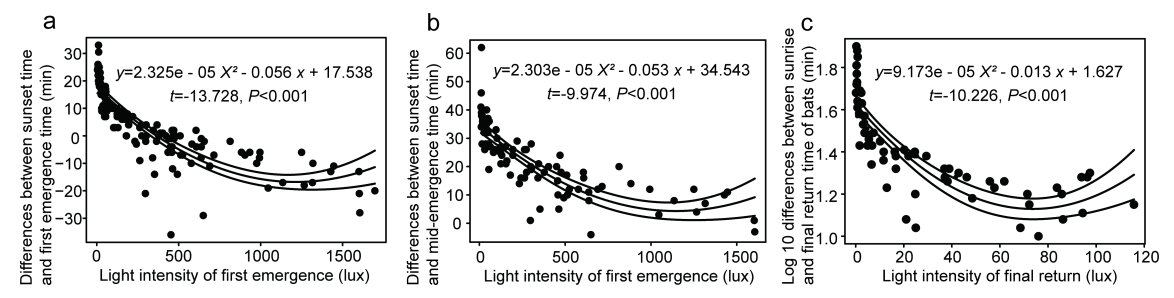

Figure 5 a: The relationship between first emergence time of bats and light intensity at the first emergence of bats, b: The relationship between mid-emergence time of bats and light intensity at the first emergence of bats; c: The relationship between final return time of bats and light intensity at the final return of bats.

\section{REFERENCES}

A.Gutierrez, E. d., F.Pessoa, V., Aguiar, L. M. S., \& M.A.Pesso, D. (2014). Effect of light intensity on food detection in captive great fruit-eating bats, Artibeus lituratus (Chiroptera: Phyllostomidae). Behavioural Processes, 109 (A), 64-69.

Acharya, P. R., Racey, P. A., McNeil, D., Sotthibandhu, S., \& Bumrungsri, S. (2015). Timing of cave emergence and return in the dawn bat (Eonycteris spelaea, Chiroptera: Pteropodidae) in Southern Thailand. Mammal Study, 40 (1), 47-52.

Anderson, D. R., \& Burnham, K. P. (2002). Avoiding pitfalls when using information-theoretic methods. The Journal of Wildlife Management, 66 (3), 912-918.

Arndt., R. J., O’Keefe., J. M., Mitchell., W. A., Holmes., J. B., \& Lima., S. L. (2018). Do predators influence the behaviour of temperate-zone bats? An analysis of competing models of roost emergence times. Animal Behaviour, 145 (2018), 161-170.

Barclay, R. M. R. (1989). The effect of reproductive condition on the foraging behavior of female hoary bats, Lasiurus cinereus. Behavioral Ecology and Sociobiology, 24 (1989), 31-37.

Bartoń., K. (2014). MuMIn: multi-model inference. R package version 1.10.5. R topics documented , 1-75.

Bell-Pedersen, D., Cassone, V. M., Earnest, D. J., Golden, S. S., Hardin, P. E., Thomas, T. L., \& Zoran, M. J. (2005). Circadian rhythms from multiple oscillators lessons from diverse organisms. Nature Reviews Genetics, 6 , 544-556. doi:10.1038/nrd1633

$10.1038 / \operatorname{nrg} 1633$

Brown, J. S. (1988). Patch use as an indicator of habitat preference, predation risk, and competitionBehavioral Ecology and Sociobiology, 22 (1), 37-47.

Bullock., D. J., Combes., B. A., Eales., L. A., \& Pritchard., J. S. (1987). Analysis of the timing and pattern of emergence of the pipistrelle bat (Pipistrellus pipistrettus ). Journal of Zoology, 211 (2), $267-274$.

Caravaggi, A., Gatta, M., Vallely, M.-C., Hogg, K., Freeman, M., Fadaei, E., . . . Tosh, D. G. (2018). Seasonal and predator-prey effects on circadian activity of free-ranging mammals revealed by camera traps. PeerJ, 6 , e5827. doi:10.7717/peerj.5827

Caro, T. (2005). Antipredator defenses in birds and mammals . Chicago: University of Chicago Press.

Chevan., A., \& Sutherland., M. (1991). Hierarchical Partitioning. The American Statistician, 45 (2), $90-96$.

Erickson, J. L., \& West, S. D. (2002). The influence of regional climate and nightly weather conditions on activity patterns of insectivorous bats. Acta Chiropterologica, 4 (1). 
Erkert, H. G. (2004). Extremely low threshold for photic entrainment of circadian activity rhythms in molossid bats (Molossus molossus; Chiroptera-Molossidae).Mammalian Biology, 69 (2004), 361-374.

Fenton, M. B., Rautenbach, I. L., Smith, S. E., Swanepoel, C. M., Grosell, J., \& Jaarsveld, J. v. (1994). Raptors and bats threats and opportunities. Animal Behaviour, 48 (1), 9-18.

Foà, A., \& Bertolucci, C. (2001). Temperature cycles induce a bimodal activity pattern in ruin lizards masking or clock-controlled event a seasonal problem. Journal of Biological Rhythms, 16 (6), 574-584.

Fox., R., Lehmkuhle., S. W., \& Westendorf., D. H. (1976). Falcon visual acuity. Science, 192 (4236), 263-265.

Frick, W. F., Stepanian, P. M., Kelly, J. F., Howard, K. W., Kuster, C. M., Kunz, T. H., \& Chilson, P. B. (2012). Climate and weather impact timing of emergence of bats.PLoS One, 7 (8), e42737. doi:10.1371/journal.pone.0042737.g001

Irwin, N. P., \& Speakman, J. R. (2003). Azorean Bats Nyctalus azoreum , Cluster as they Emerge from Roosts, Despite the Lack of Avian Predators. Acta Chiropterologica, 52 (2), 185-192.

Jin., L., Wang., J., Zhang., Z., Sun., K., Kanwal., J. S., \& Feng., J. (2012). Postnatal development of morphological and vocal features in Asian particolored bat, Vespertilio sinensis . Mammalian Biology, 77 (2012), 339-344.

John, A. (2013). Animal Behavior: An Evolutionary Approach. In A. John (Ed.), Animal Behavior: An Evolutionary Approach. Sunderland: Sinauer Associates.

Jones, G., \& Rydell, J. (1994). Foraging strategy and predation risk as factors influencing emergence time in echolocating bats. Philosophical Transactions of the Royal Society B, 346 (1318), 445-455.

Lee, Y., \& McCracken, G. F. (2001). Timing and variation in the emergence and return of Mexican free-tailed bats, Tadarida brasiliensis mexicana. Zoological Studies, 40 (4), 309-316.

LeGates, T. A., Fernandez, D. C., \& Hattar, S. (2014). Light as a central modulator of circadian rhythms, sleep and affect. Nature Reviews Neuroscience, 15 (7), 443-454.

Levy, O., Dayan, T., Porter, W. P., \& Kronfeld-Schor, N. (2018). Time and ecological resilience: can diurnal animals compensate for climate change by shifting to nocturnal activity? Ecological Monographs, 89 (1), e01334.

Lima, S. L. (1998). Stress and Decision Making under the Risk of Predation: Recent Developments from Behavioral, Reproductive, and Ecological Perspectives. Advances in the Study of Behavior, 27 , 215-290. doi:10.1016/s0065-3454(08)60366-6

Lima, S. L., \& O'Keefe, J. M. (2013). Do predators influence the behaviour of bats? Biol Rev Camb Philos Soc, 88 (3), 626-644. doi:10.1111/brv.12021

Lin., A., \& Feng, J. (2008). Solving multicollinearity in the process of fitting regression model using the nested estimate procedure. Quality 83 Quantity, 42 (3), 417-426.

Lučan, R. (2009). Effect of colony size and reproductive period on the emergence behaviour of a maternity colony of Daubenton's Bat (Myotis daubentonii ) occupying an artificial roost (Chiroptera: Vespertilionidae). Folia Parasitologica, 53 , 147-152.

McAney, C. M., \& Fairley, J. S. (1988). Habitat preference and overnight and seasonal variation in the foraging activity of lesser horseshoe bats. Acta Theriologica, 33 , 393-402.

McClung, C. R. (2000). Circadian rhythms in plants: a millennial view. Physiologia Plantarum, 109 (4), 359-371.

Mougeot, F., \& Bretagnolle, V. (2000). Predation risk and moonlight avoidance in nocturnal seabirds. Journal of Avian Biology, 31 , 376-386. 
Pavey, C. R., Burwell, C. J., Grunwald, J.-E., Marshall, C. J., \& Neuweiler, G. (2001). Dietary benefits of twilight foraging by the insectivorous batHipposideros speoris . Biotropica, 33 (4), 670-681. doi:10.1111/j.17447429.2001.tb00224.x

Pita, R., Mira, A., \& Beja, P. (2011). Circadian activity rhythms in relation to season, sex and interspecific interactions in two mediterranean voles. Animal Behaviour, 81 (5), 1023-1030.

Quaglietta, L., Mira, A., \& Boitani, L. (2018). Extrinsic and intrinsic factors affecting the daily rhythms of a semiaquatic carnivore in a mediterranean environment.Hystrix, 29 (1), 128-136.

R Core Development Team. (2018). R: a language and environment for statistical computing, 3.5.1 edn. Vienna (Austria): R Foundation for Statistical Computing. Retrieved from: http://www.R-proje ct.org .

R. A. Saldaña-Vázquez, \& Munguía-Rosas, M. A. (2013). Lunar phobia in bats and its ecological correlates: A meta-analysis. Mammalian Biology, 78 (3), 216-219.

Russo, D., LucaCistrone, \& GarethJones. (2007). Emergence time in forest bats: the influence of canopy closure. Acta Oecologica, 31 (1), 119-126.

Rydell, J., Entwistle, A., \& Racey, P. A. (1996). Timing of foraging flights of three species of bats in relation to insect activity and predation risk. Oikos, 76 (2), 243-252.

Rydell, J., \& Speakman, J. R. (1995). Evolution of nocturnality in bats: Potential competitors and predators during their early history. Biological Journal of the Linnean Society, 54 (2), 183-191. doi:10.1111/j.10958312.1995.tb01031.x

Shiel, C. B., \& Fairley, J. S. (1999). Evening emergence of two nursery colonies of Leisler's bat (Nyctalus leisleri ) in Ireland. Journal of Zoology, 247 (4), 439-447.

Shuert, C. R., Halsey, L. G., Pomeroy, P. P., \& Twiss, S. D. (2020). Energetic limits: Defining the bounds and trade-offs of successful energy management in a capital breeder. J Anim Ecol, 89 (11), 2461-2472. doi:10.1111/1365-2656.13312

Speakman, J. R. (1991). Why do insectivorous bats in Britain not fly in daylight more frequently? Functional Ecology, 5 (4), 518-524.

Speakman, J. R., Hays, G. C., \& Webb, P. I. (1994). Is hyperthermia a constraint on the diurnal activity of bats. Journal of Theoretical Biology, 171 (3), 325-339.

Tester, J. R., \& Figala, J. (1990). Effects of biological and environmental factors on activity rhythms of wild animals. Progress in Clinical and Biological Research, 341 (B), 809-819.

Voigt, C. C., \& Kingston, T. (2016). Bats in the Anthropocene: Conservation of Bats in a Changing World.Springer International Publishing .

Walsh., C., \& Nally., R. M. (2020). hier.part: Hierarchical Partitioning. $R$ topics documented , 1-13.

Wang., Y., BY Shi, X Zhao, Feng., J., \& Jiang., T. (2020). Morphological correlates of personality in female asian particolored bats (Vespertilio sinensis ). Animals, 10 (2), 1-15.

Welbergen, J. A. (2006). Timing of the evening emergence from day roosts of the grey-headed flying fox,Pteropus poliocephalus : the effects of predation risk, foraging needs, and social context. Behavioral Ecology and Sociobiology, 60 (3), 311-322.

Welbergen, J. A. (2008). Variation in twilight predicts the duration of the evening emergence of fruit bats from a mixed-species roost. Animal Behaviour, 75 (4), 1543-1550.

Yerushalmi, S., \& Green, R. M. (2009). Evidence for the adaptive significance of circadian rhythms.Ecology letters, 12 (9), 970-981. doi:10.1111/j.1461-0248.2009.01343.x 
Yin, Z. (2020). Study on Intestinal Microbiome during Development of the Asian Particolored Bat (Vespertilio sinensis). Jilin Agricultural University, 